Pacific

Journal of

Mathematics

DIFFERENTIAL GEOMETRY OF GENERALIZED SUBMANIFOLDS

Silvano Delladio

Volume $194 \quad$ No. 2

June 2000 


\title{
DIFFERENTIAL GEOMETRY OF GENERALIZED SUBMANIFOLDS
}

\author{
Silvano Delladio
}

\begin{abstract}
The curvature of a surface can be recovered from the tangent space to the graph of the Gauss map. Exploiting this observation we manage to equip a generalized Gauss graph with the standard tools of differential geometry: Weingarten map, second fundamental form, Riemann curvature tensor. Several variational applications are given.
\end{abstract}

\section{Introduction and outline of the results.}

In the recent past a lot of authors have devoted systematic efforts to indagate problems related to compactness and degeneration phenomenons in classes of surfaces with integral bounds on curvature, both on the front of geometric measure theory and on that of differential geometry. As a general reference reviewing the main results obtained in this area by differential geometers, we refer the reader to [1] while, unfortunately, we have no paper so efficaciously summarizing the theory developed in the setting of geometric measure theory to recommend.

These two points of view are deeply different and only a few attempts to bridge the gap existing between them have been done up to now. As very interesting examples of steps in this direction from geometric measure theory, we have to mention the papers about curvature varifolds by Hutchinson ([19], [20], [21], [22]) and Mantegazza $([\mathbf{2 4}])$ where a notion of generalized second fundamental form is defined and applied to variational problems. We also have to recall the papers on generalized Gauss graphs ([2], [3], [5], [10], $[\mathbf{1 1}],[\mathbf{1 2}],[\mathbf{1 3}],[\mathbf{1 4}],[\mathbf{1 5}])$. In particular, in paper [14], special generalized Gauss graphs have been used to prove a result of differential geometry ([14, Theorem 7.1]) which generalizes a theorem by Langer [23].

As far as this paper is concerned, our main goal will be to equip generalized Gauss graphs with the standard tools of differential geometry (Weingarten map, second fundamental form, Riemann curvature tensor), coherently extended, in such a way that they continue to have nice properties and satisfy the usual relations, useful to perform geometric calculations. We believe that this machinery could be helpful in pursuing the "bridge project" 
mentioned above, making simpler a comparison between results from differential geometry and results from geometric measure theory.

The idea from which we proceed characterizes Section $\S 3$ and it can be summarized as follows: The curvature of an oriented smooth surface at a given point can be recovered from the tangent space of the Gauss graph, i.e., the graph of the Gauss map, at the corresponding point. As a consequence of this simple observation, one is led in a natural way to look for a formula expressing the Weingarten map through the multivector orienting the Gauss graph. Hence we arrive to define the generalized Weingarten map, that is the Weingarten map associated to a generalized Gauss graph (Definition 5.2 ), and to prove that it is self-adjoint just as in the smooth case. Then generalized principal directions and curvatures can be defined, and the usual linear properties of the Riemann curvature tensor can be extended easily to this generalized setting (Proposition 5.2).

Section $\S 4$ is devoted to collecting some well known facts about generalized Gauss graphs, going into some aspects which, otherwise, could be incomprehensible for a reader unnaccustomed to this subject.

In Section $\S 6$ we compute the length of strata of the multivector orienting a generalized Gauss graph and, as a consequence, we get an estimate of the length of a fixed stratum by means of the length of a lower order stratum (Proposition 6.1). Such a result has been applied in Section $\S 7$ where we deduce an inequality (Proposition 7.5) useful for solving variational problems involving special generalized Gauss graphs. Indeed the estimated functional $\mathcal{G}_{F_{q}}$ has been proved in [13] to be cohercive enough to allow compactness in the class of special generalized Gauss graphs. Proposition 7.6 implies, in particular, that a conjecture stated in $[\mathbf{1 4}]$ is true.

In Proposition 7.1 we prove that, for codimension 1 special generalized Gauss graphs, the integral of the greatest among the nonnegative parts of principal curvatures is lower semicontinuous with respect to the weak convergence of currents. Moreover, in view of future works involving integrals of curvatures, we prove the measurability of the symmetric functions of principal curvatures with respect to a normal vector field (Proposition 7.3).

In the last section, $\S 8$, we have collected some variational applications of the theory developed in this paper and in the previous ones [12], [13], [14].

\section{General notation.}

For the general terminology we refer to the classical literature about geometric measure theory (see [18], [26], [28]) and about differential geometry (e.g., [9], [16], [17]). In regard to the classical measure theory, a completely standard terminology will be adopted (e.g., [27]).

Rectifiable currents are the main tools we will work with. Let $U$ be an open subset of a given euclidean space. Then, according to a completely 
standard notation, the set of smooth differential $h$-forms having compact support in $U$ will be denoted by $\mathcal{D}^{h}(U)$, while $\mathcal{D}_{h}(U)$ will be the set of $h$-dimensional currents in $U$. For the usual mass of a current $T$ we use the notation $\mathbf{M}(T)$. More generally, if $W$ is any open subset of $U$, then we define $\mathbf{M}_{W}(T)$ according to 26.5 in [28].

We will also need the class $\mathbf{I}_{h}(U)$ of integral currents, i.e., the set of $T \in \mathcal{D}_{h}(U)$ such that $T$ is an integer multiplicity current and

$$
\mathbf{M}_{W}(T)+\mathbf{M}_{W}(\partial T)<+\infty
$$

for all $W \subset \subset U$. Note that, by Boundary Rectifiability Theorem, $\partial T$ is an integer multiplicity current whenever $T \in \mathbf{I}_{h}(U)$.

According to Definition 4.1 below, a generalized Gauss graph is an integral current satisfying certain relations trivially fulfilled by the graph of the Gauss map to a smooth oriented surface viewed as a current. Dimension and codimension of the surface will be denoted by $n$ and $k$. The euclidean spaces we need to introduce are the following

$$
\mathbf{R}_{x}^{N}, \quad \Lambda^{k} \mathbf{R}_{x}^{N} \cong \mathbf{R}_{y}^{d}, \quad \mathbf{R}_{x}^{N} \times \Lambda^{k} \mathbf{R}_{x}^{N} \cong \mathbf{R}_{x}^{N} \times \mathbf{R}_{y}^{d}
$$

where

$$
N:=n+k, \quad d:=\left(\begin{array}{c}
N \\
k
\end{array}\right)
$$

and the subscripts specify the variable names.

The standard basis (resp. dual basis) of $\mathbf{R}_{x}^{N}$ (resp. $\left(\mathbf{R}_{x}^{N}\right)^{\prime} \cong \mathbf{R}_{x}^{N}$ ) will be denoted by $e_{1}, \ldots, e_{N}$ (resp. $d x^{1}, \ldots, d x^{N}$ ) while $\left\{\varepsilon_{\beta}\right\}_{\beta \in I(N, k)}$ will be the standard basis of $\mathbf{R}_{y}^{d}$.

To simplify the formulae, throughout the paper we will often omit mention of the obvious isometric isomorphism

$$
\Phi: \Lambda^{k} \mathbf{R}_{x}^{N} \rightarrow \mathbf{R}_{y}^{d} \quad\left(\Phi\left(e_{\beta_{1}} \wedge \cdots \wedge e_{\beta_{k}}\right)=\varepsilon_{\beta}\right)
$$

and immersions

$$
\mathbf{R}_{x}^{N} \hookrightarrow \mathbf{R}_{x}^{N} \times \mathbf{R}_{y}^{d}, \quad \mathbf{R}_{y}^{d} \hookrightarrow \mathbf{R}_{x}^{N} \times \mathbf{R}_{y}^{d} .
$$

Furthermore, for the convenience of the reader, we set also

$$
[\cdot]^{\bullet}:=\Phi, \quad[\cdot]_{\bullet}:=\Phi^{-1}
$$

e.g., $\left[e_{\beta_{1}} \wedge \cdots \wedge e_{\beta_{k}}\right]^{\bullet}=\varepsilon_{\beta}$ and $\left[\varepsilon_{\beta}\right]_{\bullet}=e_{\beta_{1}} \wedge \cdots \wedge e_{\beta_{k}}$.

Given a couple of positive integers $H$ and $h$ such that $H \geq h \geq 0$, we adopt the following notation for multi-indices sets:

$$
I(H, h):= \begin{cases}\left\{\delta=\left(\delta_{1}, \ldots, \delta_{h}\right) \mid 1 \leq \delta_{1}<\delta_{2}<\ldots<\delta_{h} \leq H\right\} & \text { if } h>0 \\ \emptyset & \text { if } h=0\end{cases}
$$


If $\alpha \in I(H, h)$, then $\sigma(\alpha, \bar{\alpha})$ is the sign of the permutation of $(1,2, \ldots, H)$ into $(\alpha, \bar{\alpha})$. The short notation for wedge products will often be used. For example:

$$
e_{\alpha}:=e_{\alpha_{1}} \wedge \ldots \wedge e_{\alpha_{n}} \quad \text { or } \quad d x^{\beta}:=d x^{\beta_{1}} \wedge \ldots \wedge d x^{\beta_{k}} .
$$

In order to distinguish the wedge and interior products in $\mathbf{R}_{x}^{N} \times \mathbf{R}_{y}^{d}$ from the same operations in $\mathbf{R}_{x}^{N}$, the first ones will be indicated by

$$
\mathbb{A} \text { and } \mathbb{L}
$$

respectively.

The projection operator from the product space $\mathbf{R}_{x}^{N} \times \mathbf{R}_{y}^{d}$ onto the first factor space $\mathbf{R}_{x}^{N}$ will be denoted $\mathbf{p}$. Given a linear transformation $L$, we shall use the so-called "Hilbert-Schmidt norm" of $L$, i.e.,

$$
|L|:=\sqrt{\operatorname{trace}\left(L^{t} \circ L\right)} .
$$

Given a nonzero simple multivector $\tau$, the corresponding linear subspace $E_{\tau}$ will be called enveloping subspace of $\tau$ (according to [25]).

Both the standard inner product and the dual pairing for all the vector spaces we will deal with will be denoted by $\langle\cdot, \cdot\rangle$. For example, if $\eta, \tau \in$ $\Lambda^{n}\left(\mathbf{R}_{x}^{N}\right)$,

$$
\langle\eta, \tau\rangle:=\sum_{\alpha}\left\langle\eta, e_{\alpha}\right\rangle\left\langle\tau, e_{\alpha}\right\rangle=\sum_{\alpha} \eta^{\alpha} \tau^{\alpha} .
$$

The only notion of length we will need is the natural one induced by $\langle\cdot, \cdot\rangle$, namely:

$$
|\cdot|:=\sqrt{\langle\cdot, \cdot\rangle}
$$

It is convenient to introduce a notation also for the set of unit vectors in $\mathbf{R}_{y}^{d}$ : Let it be denoted $\mathbf{S}_{1}\left(\mathbf{R}_{y}^{d}\right)$. For $h=0,1, \ldots, n$, the $h$-th stratum of a vector

$$
\zeta \in \Lambda^{n}\left(\mathbf{R}_{x}^{N} \times \mathbf{R}_{y}^{d}\right)
$$

is defined as follows:

$$
\zeta_{(h)}:=\sum_{\substack{\gamma \in I(N, n-h) \\ \delta \in I(N, h)}}\left\langle\zeta, e_{\gamma} \wedge \varepsilon_{\delta}\right\rangle e_{\gamma} \wedge \varepsilon_{\delta} .
$$

Analogously one can define the strata $\omega_{(h)}$ of a form $\omega \in \mathcal{D}^{n}\left(\mathbf{R}_{x}^{N} \times \mathbf{R}_{y}^{d}\right)$ and hence, in the natural way, also the strata of a current can be defined. For example, if $T=\llbracket G, \eta, \theta \rrbracket$ is an integer multiplicity rectifiable $n$-current in $\mathbf{R}_{x}^{N} \times \mathbf{R}_{y}^{d}$ and $h \in\{0,1, \ldots, n\}$, then we set

$$
T_{(h)}(\omega):=T\left(\omega_{(h)}\right)=\int_{G}\left\langle\eta_{(h)}, \omega\right\rangle \theta d \mathcal{H}^{n}
$$


for all $\omega \in \mathcal{D}^{n}\left(\mathbf{R}_{x}^{N} \times \mathbf{R}_{y}^{d}\right)$. Obviously the strata $T_{(h)}$, as the current $T$, can be regarded as vector measures and then (2.1) implies that

$$
\left|T_{(h)}\right|=\left|\eta_{(h)}\right| \theta \mathcal{H}^{n}\left\llcorner G=\left|\eta_{(h)}\right||T|\right.
$$

where $|\cdot|$ denotes, in this case, the total variation.

Moreover, with reference to the same rectifiable current, we set also:

$$
G^{*}:=\left\{(x, y) \in G \mid \eta_{(0)}(x, y) \neq 0\right\} .
$$

Finally we will adopt the standard inclusion and equality symbols to denote the corresponding notions in measure. For example, if $A$ and $B$ are $\mathcal{H}^{h}$-measurable sets, then " $A \subset B$ " will mean $\mathcal{H}^{h}(A \backslash B)=0$.

Further notations and conventions will be introduced in each section below, if need be.

\section{Some preliminary results about smooth submanifolds of $\mathbf{R}_{x}^{N}$.}

Let $M$ be a $n$-dimensional smooth submanifold of $\mathbf{R}_{x}^{N}$, oriented by a smooth field $\tau$ of unit simple $n$-vectors tangent to $M$.

Define

$$
\nu:=[* \tau]^{\bullet}, \quad \xi:=\mathbb{A}^{n}(I \oplus d \nu) \tau
$$

and note that $\xi$ is a simple $n$-vector field, tangent to the graph of $\nu$. Note also that $\xi_{(0)}=\tau$.

Since the curvature tensor is completely determined by $d \nu$, we expect a lot of information about curvature could be easily deduced from $\xi$. According to this expectation, in the first subsection below we show how to recover the Weingarten map of $M$ from $\xi$, while, in the second subsection, we get the expression of $\xi$ in terms of principal curvatures. These results will allow to generalize the notion of curvature and to interpret integral functionals involving curvatures as energies in the context of generalized Gauss graphs.

3.1. Weingarten map through $\xi$. Given $p \in M$ and $w \in\left[M_{p}^{\perp}\right]^{\bullet}$, let

$$
A_{M, p}^{w}: M_{p} \rightarrow M_{p}
$$

denote the classical Weingarten map of $M$ at $p$ with respect to $w$ (e.g., see $[\mathbf{9}]$ ). Then the following proposition holds.

Proposition 3.1. One has

$$
A_{M, p}^{w}(v)=(-1)^{k+n+1}\left[\xi _ { ( 1 ) } ( p ) \amalg ( \tau ( p ) \llcorner v ) ] \bullet \left\llcorner\left([\nu(p)] \bullet\left\llcorner w_{\bullet}\right) .\right.\right.\right.
$$

for all $v \in M_{p}$.

Proof. Obviously, we can assume $|w|=1$. Let $\left\{\nu_{1}, \ldots, \nu_{k}\right\}$ be an orthonormal family of unit vector fields, defined and of class $C^{1}$ close to $p$ on $M$, such that

$$
\nu_{1}(p)=w_{\bullet} \quad \text { and } \quad\left[\nu_{1} \wedge \cdots \wedge \nu_{k}\right]^{\bullet}=\nu .
$$


Then, at the point $p$, we have

$$
\begin{aligned}
{[d \nu(v)]_{\bullet} } & =\sum_{j}(-1)^{j-1} d \nu_{j}(v) \wedge \nu_{\bar{j}} \\
& =\sum_{j}(-1)^{j-1}\left(d \nu_{j}(v)\right)^{T} \wedge \nu_{\bar{j}}+\sum_{j}(-1)^{j-1}\left\langle d \nu_{j}(v) \mid \nu_{j}\right\rangle \nu_{j} \wedge \nu_{\bar{j}} \\
& =\sum_{j}(-1)^{j} A_{M}^{\nu_{j}^{\bullet}}(v) \wedge \nu_{\bar{j}}
\end{aligned}
$$

whence we obtain

$$
\begin{aligned}
{\left[d \nu_{p}(v)\right]_{\bullet}\left\llcorner\left([\nu(p)]_{\bullet}\left\llcorner w_{\bullet}\right)\right.\right.} & =\left[d \nu_{p}(v)\right] \bullet\left\llcorner\nu_{\overline{1}}(p)\right. \\
& =-\left[A_{M, p}^{\nu_{1}^{\bullet}}(v) \wedge \nu_{\overline{1}}(p)\right]\left\llcorner\nu_{\overline{1}}(p)\right. \\
& =(-1)^{k} A_{M, p}^{w}(v) .
\end{aligned}
$$

Now, if $\left\{\tau_{h}\right\}$ is an orthonormal basis of $M_{p}$, then one has

$$
\xi_{(1)}(p)=\left[\mathbb{\aleph}^{n}\left(I \oplus d \nu_{p}\right) \tau(p)\right]_{(1)}=\sum_{h}(-1)^{h-1} d \nu_{p}\left(\tau_{h}\right) \mathbb{A} \tau_{\bar{h}}
$$

which implies

$$
\xi_{(1)}(p) \llbracket \tau_{\bar{h}}=(-1)^{n+h} d \nu_{p}\left(\tau_{h}\right)
$$

i.e.,

$$
\xi_{(1)}(p) \longleftarrow\left(\tau(p)\left\llcorner\tau_{h}\right)=(-1)^{n+1} d \nu_{p}\left(\tau_{h}\right)\right.
$$

and then also

$$
\xi_{(1)}(p) \longleftarrow\left(\tau(p)\llcorner v)=(-1)^{n+1} d \nu_{p}(v) .\right.
$$

The conclusion follows by (3.3).

3.2. Expression of $\xi$ in terms of curvature. Before proceeding to the computation in any dimension and codimension, let us consider the following example concerning the case of a curve in three dimensional space.

Example $3.1(\mathrm{n}=2, \mathrm{k}=1)$. Let $M$ be a curve in $\mathbf{R}_{x}^{3}$ and consider the Frenet frame $\{\tau, \mathbf{n}, \mathbf{b}\}$ along $M$ (see $[\mathbf{1 6}, \S 1-5])$. If $\kappa_{n}$ and $\kappa_{b}$ denote the principal curvatures of $M$ with respect to $\mathbf{n}$ and $\mathbf{b}$, i.e., the eigenvalues of $A_{M}^{\mathbf{n}^{\bullet}}$ and $A_{M}^{\mathbf{b}^{\bullet}}$ respectively, then formula (3.2) above yields

$$
d \nu(\tau)=\left[-A_{M}^{\mathbf{n}^{\bullet}}(\tau) \wedge \mathbf{b}+A_{M}^{\mathbf{b}^{\bullet}}(\tau) \wedge \mathbf{n}\right]^{\bullet}=-\kappa_{n}(\tau \wedge \mathbf{b})^{\bullet}+\kappa_{b}(\tau \wedge \mathbf{n})^{\bullet} .
$$

Hence

$$
\xi=\tau+d \nu(\tau)=\tau-\kappa_{n}(\tau \wedge \mathbf{b})^{\bullet}+\kappa_{b}(\tau \wedge \mathbf{n})^{\bullet}
$$

i.e.,

$$
\xi_{(0)}=\tau, \quad \xi_{(1)}=-\kappa_{n}(\tau \wedge \mathbf{b})^{\bullet}+\kappa_{b}(\tau \wedge \mathbf{n})^{\bullet}
$$


The general case is rather complicated and requires a supplement of notation. First of all, set

$$
J:=\{1, \ldots, k\} \times\{1, \ldots, n\}=\{(j, l) \mid 1 \leq j \leq k, 1 \leq l \leq n\},
$$

and define

$$
X_{h}^{*}:=\left\{\left(\gamma_{1}, \ldots, \gamma_{h}\right) \in J^{h} \mid \gamma_{i} \neq \gamma_{j} \text { whenever } i \neq j\right\}
$$

where $h \in\{1, \ldots, n\}$. Then consider the following equivalence relation on $X_{h}^{*}$ :

$$
\Gamma_{1} \sim \Gamma_{2} \quad \text { if } \Gamma_{2} \text { is a permutation of } \Gamma_{1}
$$

and choose a complete set of representatives from the equivalence classes, which will be denoted by $X_{h}$.

Now consider an orthonormal basis $\left\{w_{j}\right\}$ of $E_{[\nu(p)} \bullet \bullet$ Then for all $j$ one can find the generalized principal curvatures $\kappa_{l}\left(w_{j}\right)$ of $M$ at $p$ with respect to $w_{j}$, i.e., the eigenvalues of $A_{M, p}^{w_{j}}$, and the corresponding principal directions $\tau_{l}\left(w_{j}\right)$.

For $\gamma=(j, l) \in J$ let us set

$$
\tau_{\gamma}:=\tau_{l}\left(w_{j}\right), \quad \kappa_{\gamma}:=\kappa_{l}\left(w_{j}\right) .
$$

Consider an orthonormal basis $\left\{u_{j}\right\}$ of $E_{\tau(p)}$ and $\alpha \in I(n, h)$. If for a given $\Gamma=\left(\gamma_{1}, \ldots, \gamma_{h}\right) \in X_{h}^{*}$ we define

$$
\tau_{\Gamma}:=\tau_{\gamma_{1}} \wedge \cdots \wedge \tau_{\gamma_{h}}
$$

then we recall that one has

$$
\left\langle\tau_{\Gamma} \mid u_{\alpha}\right\rangle=\operatorname{det}\left(\left\langle\tau_{\gamma_{r}} \mid u_{\alpha_{s}}\right\rangle\right)_{r, s}
$$

i.e.,

$$
\left\langle\tau_{\Gamma} \mid u_{\alpha}\right\rangle=\sum_{\substack{\Gamma^{\prime} \sim \Gamma \\ \Gamma^{\prime}=\left(\gamma_{1}^{\prime}, \cdots, \gamma_{h}^{\prime}\right)}} \sigma\left(\Gamma^{\prime}, \Gamma\right)\left\langle\tau_{\gamma_{1}^{\prime}} \mid u_{\alpha_{1}}\right\rangle \cdots\left\langle\tau_{\gamma_{h}^{\prime}} \mid u_{\alpha_{h}}\right\rangle
$$

where $\sigma\left(\Gamma^{\prime}, \Gamma\right)$ denotes the signature of the permutation mapping $\Gamma^{\prime}$ to $\Gamma$. Finally, the first component of $\gamma \in J$ will be indicated by $j_{\gamma}$, while

$$
\kappa_{\Gamma}:=\kappa_{\gamma_{1}} \cdots \kappa_{\gamma_{h}}
$$

for all given $\Gamma=\left(\gamma_{1}, \ldots, \gamma_{h}\right) \in X_{h}^{*}$.

Now we are ready to state the expression of $\xi$ in terms of principal curvatures. Indeed, the definition (3.1) of $\xi$ implies immediately

$$
\xi_{(h)}=\sum_{\alpha \in I(n, h)} \sigma(\alpha, \bar{\alpha})\left[\left(\mathbb{\wedge}^{h} d \nu\right) u_{\alpha}\right] \mathbb{\wedge} u_{\bar{\alpha}}
$$


for $h=1, \ldots, n$, and it can be computed that

$$
\begin{aligned}
& \left(\mathbb{A}^{h} d \nu\right) u_{\alpha}
\end{aligned}
$$

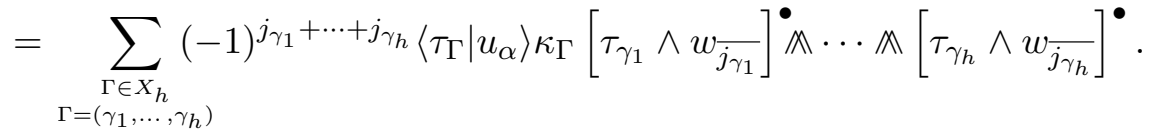

Hence it follows

$$
\left|\xi_{(h)}\right|^{2}=\sum_{\Gamma \in X_{h}} \kappa_{\Gamma}^{2}\left|\tau_{\Gamma}\right|^{2}, \quad|\xi|^{2}=1+\sum_{h=1}^{n} \sum_{\Gamma \in X_{h}} \kappa_{\Gamma}^{2}\left|\tau_{\Gamma}\right|^{2} .
$$

In particular, note that $\left|\xi_{(1)}\right|$ coincides with the length of the second fundamental form of $M$ (see $[\mathbf{6}, \S 29.4 .2])$.

As we shall see, these formulas and the argument necessary to prove them hold also in the context of generalized Gauss graphs. This is the reason why we prefer to postpone the proof to Section $\S 6$ below.

If $M$ is a hypersurface, i.e., $k=1$, the set $\left\{w_{j}\right\}$ consists of a single element. Then the corresponding principal curvatures and directions can be simply denoted by $\left\{\kappa_{j}\right\}$ and $\left\{\tau_{j}\right\}$. Taking $u_{j}=\tau_{j}$, the previous formulas become

$$
\xi_{(h)}(x)=(-1)^{h} \sum_{\alpha \in I(n, h)} \sigma(\alpha, \bar{\alpha}) \kappa_{\alpha} \tau_{\alpha}^{\bullet} \mathbb{A} \tau_{\bar{\alpha}}
$$

for $h=1, \ldots, n$, and

$$
\left|\xi_{(h)}\right|^{2}=\sum_{\alpha \in I(n, h)} \kappa_{\alpha}^{2}, \quad|\xi|^{2}=1+\sum_{h=1}^{n} \sum_{\alpha \in I(n, h)} \kappa_{\alpha}^{2}
$$

where

$$
\kappa_{\alpha}:=\kappa_{\alpha_{1}} \cdots \kappa_{\alpha_{h}}, \quad \tau_{\alpha}^{\bullet}:=\tau_{\alpha_{1}}^{\bullet} \mathbb{A} \cdots \mathbb{A} \tau_{\alpha_{h}}^{\bullet} .
$$

As an interesting subcase we have

$$
\left|\xi_{(2)}\right|=\frac{|R|}{2} \quad(R \text { is the Riemann tensor of } M)
$$

which will be proved below, in the generalized setting, along with the previous identities.

\section{Generalized Gauss graphs.}

Quite a lot of results about generalized Gauss graphs have been obtained since they were defined in [5]. In this section, we recall just some basic definitions and simple facts necessary to understand the statements in the propositions of next sections. For a more exhaustive presentation of generalized Gauss graphs, their applications and relations with other subjects (e.g., curvature varifolds), we refer to papers $[\mathbf{2}],[\mathbf{3}],[\mathbf{4}],[\mathbf{5}],[\mathbf{1 0}],[\mathbf{1 1}],[\mathbf{1 2}]$, $[\mathbf{1 3}],[\mathbf{1 4}]$ and $[\mathbf{1 5}]$. 
4.1. Basic definitions. The notion of generalized Gauss graph was introduced at first in the particular case of codimension 1 (see [5, Definition 2.7]) and then for general codimension (see [11, Definition 3.1]). Special generalized Gauss graphs were defined in [13, Definition 4.1] where we applied them to solve variational problems. The class of generalized Gauss graphs can be graded into subclasses with nice properties, the smallest of which is just the set of special generalized Gauss graphs (see [14]).

Definition 4.1 ([5], [11]). Let $\Omega$ be an open subset of $\mathbf{R}_{x}^{N}$. Then we define $\operatorname{curv}_{\mathrm{n}}(\Omega)$ as the set of the currents $T \in \mathbf{I}_{n}\left(\Omega \times \mathbf{R}_{y}^{d}\right)$ such that:

(i) $T$ and $\partial T$ are carried by rectifiable subsets of $\Omega \times \mathbf{S}_{1}\left(\mathbf{R}_{y}^{d}\right)$;

(ii) $\left\langle T\left\llcorner\lambda, \varphi\llcorner\omega\rangle=0\right.\right.$ for all $\lambda \in \mathcal{D}^{n-1}\left(\Omega \times \mathbf{R}_{y}^{d}\right)$ and $\omega \in \mathcal{D}^{k-1}\left(\Omega \times \mathbf{R}_{y}^{d}\right)$, where $\varphi(x, y):=\sum_{\beta \in I(N, k)} y^{\beta} d x^{\beta}$;

(iii) $\left\langle\partial T\left\llcorner\mu, \varphi\llcorner\omega\rangle=0\right.\right.$ for all $\mu \in \mathcal{D}^{n-2}\left(\Omega \times \mathbf{R}_{y}^{d}\right)$ and $\omega \in \mathcal{D}^{k-1}\left(\Omega \times \mathbf{R}_{y}^{d}\right)$; (iv) $(-1)^{k n}\left\langle T\llcorner * \varphi, g\rangle \geq 0\right.$ for all nonnegative $g \in C_{c}\left(\Omega \times \mathbf{R}_{y}^{d}\right)$, where $*$ is the Hodge operator in $\mathbf{R}_{x}^{N}$ with respect to the canonical basis, so that $* \varphi(x, y)=\sum_{\beta \in I(N, k)} \sigma(\beta, \bar{\beta}) y^{\beta} d x^{\bar{\beta}}$.

A current belonging to $\operatorname{curv}_{\mathrm{n}}(\Omega)$ will be called generalized Gauss graph.

Definition 4.2 ([13]). A generalized Gauss graph $T$ is called special if

$$
|T| \ll\left|T_{(0)}\right| \text {. }
$$

The set of special generalized Gauss graphs belonging to $\operatorname{curv}_{n}(\Omega)$ is denoted by $\operatorname{curv}_{\mathrm{n}}^{*}(\Omega)$.

Remark 4.1. The geometrical meaning of Definition 4.1 has been discussed in [11], from which we recall some facts now. By virtue of the natural isomorphism between multivectors and multicovectors, it is easy to interpret axioms (ii)-(iii) as "orthogonality conditions" and (iv) as an "orientation condition". Indeed, if $T=\llbracket G, \eta, \theta \rrbracket \in \operatorname{curv}_{\mathrm{n}}(\Omega)$, then a standard localization argument shows that (ii) is equivalent to

$$
\left\langle\eta ( x , y ) \left\llcorner A \mid y_{\bullet}\llcorner B\rangle=0\right.\right.
$$

for all $A \in \Lambda^{n-1}\left(\mathbf{R}_{x}^{N} \times \mathbf{R}_{y}^{d}\right)$ and $B \in \Lambda^{k-1}\left(\mathbf{R}_{x}^{N}\right)$, at $\mathcal{H}^{n}\llcorner G$ almost all $(x, y)$. But this is clearly equivalent to

$$
\mathbf{p}\left(E_{\eta(x, y)}\right) \perp E_{y \bullet}
$$

at $\mathcal{H}^{n}\llcorner G$ almost all $(x, y)$. Analogously, axiom (iii) is equivalent to

$$
\mathbf{p}\left(E_{\zeta(x, y)}\right) \perp E_{y \bullet}
$$

at $\mathcal{H}^{n}\llcorner S$ almost all $(x, y)$, where $S$ and $\zeta$ denote the carrier and the orientation of $\partial T$, respectively. As for (iv), the same argument shows that it is equivalent to

$$
\left\langle * \eta_{(0)}(x, y) \mid y_{\bullet}\right\rangle \geq 0
$$


at $\mathcal{H}^{n}\llcorner G$ almost all $(x, y)$. By using (4.1) and (4.3), one can easily verify that

$$
\frac{\eta_{(0)}(x, y)}{\left|\eta_{(0)}(x, y)\right|}=(-1)^{k n} * y \bullet
$$

for $\left|T_{(0)}\right|$ almost all $(x, y)$, [11, Proposition 4.1]. Finally, note that the absolute continuity condition characterizing Definition 4.2 says that ap $D\left(p_{\mid G}\right)$ has rank $n$, i.e., the tangent space for $G$ does not contain purely $y$-directions, at $\mathcal{H}^{n}$ almost all points of $G$ (compare [13, Proposition 7.1]).

Example 4.1 (Generalized Gauss graphs induced by a smooth submanifold). Let $M$ be a $n$-dimensional surface, embedded in $\mathbf{R}_{x}^{N}$, smooth (enough), oriented by a smooth field $\tau$ of unit simple $n$-vectors tangent to $M$ and with smooth boundary $\partial M \subset M$. Then, by considering $\nu$ and $\xi$ as in (3.1), one can define

$$
G:=\text { graph of } \nu, \quad \eta:=\frac{\xi}{|\xi|} \circ \mathbf{p}
$$

and verify that

$$
\llbracket G, \eta, 1 \rrbracket \in \operatorname{curv}_{\mathrm{n}}^{*}\left(\mathbf{R}_{x}^{N}\right) .
$$

Indeed Equations (4.1) and (4.2) are verified by virtue of the obvious reciprocal orthogonality between the tangent space and the normal space, at all points of $M$. Moreover (4.3) follows at once from

$$
\eta_{(0)}=\frac{\xi_{(0)}}{|\xi|} \circ \mathbf{p}=\frac{\tau}{|\xi|} \circ \mathbf{p}
$$

which implies also the absolute continuity condition in Definition 4.2. Finally, note that the expression of $\eta$ in explicit terms of principal curvatures can be immediately obtained by the formulas of subsection $\S 3.2$.

4.2. Functionals. Given any nonnegative continuous function

$$
F(x, y ; \xi):\left(\Omega \times \mathbf{R}_{y}^{d}\right) \times \Lambda^{n}\left(\Omega \times \mathbf{R}_{y}^{d}\right) \rightarrow \mathbf{R}
$$

we can define a functional

$$
\mathcal{G}_{F}: \operatorname{curv}_{\mathrm{n}}(\Omega) \rightarrow \overline{\mathbf{R}}
$$

as follows (if $T=\llbracket G, \eta, \theta \rrbracket)$ :

$$
\begin{aligned}
\mathcal{G}_{F}(T): & =\int F\left(x, y ; \frac{\eta}{\left|\eta_{(0)}\right|}(x, y)\right) d\left|T_{(0)}\right|(x, y) \\
& =\int_{G^{*}} F\left(x, y ; \frac{\eta}{\left|\eta_{(0)}\right|}(x, y)\right)\left|\eta_{(0)}(x, y)\right| \theta(x, y) d \mathcal{H}^{n}(x, y) .
\end{aligned}
$$

Integrands we are particularly interested in are given by

$$
F_{p}^{(h)}(x, y ; \xi):=\left|\xi_{(h)}\right|^{p} \quad \text { and } \quad F_{p}:=\sum_{h=0}^{n} F_{p}^{(h)}
$$


where $p \geq 0$. Let us set

$$
E_{p}^{(h)}:=\mathcal{G}_{F_{p}^{(h)}}
$$

and note that

$$
E_{p}^{(0)}(T)=\left\|T_{(0)}\right\|:=\left|T_{(0)}\right|\left(\Omega \times \mathbf{R}_{y}^{d}\right) \quad \text { and } \quad \mathcal{G}_{F_{p}}=\sum_{h=0}^{n} E_{p}^{(h)} .
$$

If $p \geq 1$ then one can easily verify that

$$
\mathcal{G}_{F_{p}}(T) \geq C|T|\left(G^{*}\right)
$$

where $C$ is a suitable positive constant, independent from $T$. In particular, we get

$$
\mathcal{G}_{F_{p}}(T) \geq C \mathbf{M}(T)
$$

for every special generalized Gauss graph $T$ (see [13, Remark 4.4]).

Recalling $\S 3$ and more precisely the formulas obtained in subsection $\S 3.2$, we see that for the generalized Gauss graph $T$ associated to an oriented smooth hypersurface $M$ (according to Example 4.1) one has

$$
E_{p}^{(0)}(T)=\mathcal{H}^{n}(M)
$$

and

$$
E_{p}^{(h)}(T)=\int_{M}\left(\sum_{\Gamma \in X_{h}} \kappa_{\Gamma}^{2}\left|\tau_{\Gamma}\right|^{2}\right)^{\frac{p}{2}} d \mathcal{H}^{n} \quad(h=1,2, \ldots, n) .
$$

In particular, by the identities obtained in $\S 3.2$, one has

$$
E_{p}^{(1)}(T)=\int_{M} L^{p} d \mathcal{H}^{n}
$$

where $L$ is the length of the second fundamental form of $M$, and (if $k=1$ )

$$
E_{p}^{(2)}(T)=2^{-p} \int_{M}|R|^{p} d \mathcal{H}^{n}
$$

where $R$ is the Riemann tensor of $M$. In the next sections we will define the Weingarten map and the Riemann curvature tensor corresponding to a generalized Gauss graph and we will find that, even in such a generalized setting, the equalities (4.4) and (4.5) continue to hold (see Proposition 5.3 and Proposition 6.1).

\section{Generalized notions of Weingarten map, second fundamental form and Riemann curvature tensor.}

Let $T=\llbracket G, \eta, \theta \rrbracket \in \operatorname{curv}_{\mathrm{n}}\left(\mathbf{R}_{x}^{N}\right)$. Then, on the basis of Proposition 3.1, we are naturally led to consider a linear transformation

$$
\tilde{A}_{T,(x, y)}^{w}: \mathbf{R}_{x}^{N} \rightarrow \mathbf{R}_{x}^{N}
$$


for almost every $(x, y) \in G^{*}$ and for $w \in \mathbf{R}_{x}^{N}$, defined by

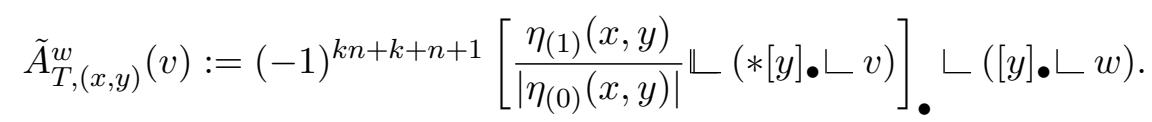

In particular, when $T$ is the Gauss graph current associated to a smooth submanifold $M$ of $\mathbf{R}^{N}$, oriented by a smooth normal field $\nu$, one has

$$
\left.\tilde{A}_{T,(p, \nu(p))}^{w}\right|_{M_{p}}=A_{M, p}^{w}
$$

at every $p \in M$ and for $w \in M_{p}^{\perp}$, by Proposition 3.1. This remark and the nice properties proved in Proposition 5.1 below, motivate the introduction of a generalized notion of Weingarten operator.

Definition 5.1. Given $T=\llbracket G, \eta, \theta \rrbracket \in \operatorname{curv}_{\mathrm{n}}\left(\mathbf{R}_{x}^{N}\right)$, let us define $\xi_{T}(x, y):=\frac{\eta(x, y)}{\left|\eta_{(0)}(x, y)\right|} \quad$ and $\quad \tau_{T}(x, y):=\frac{\eta_{(0)}(x, y)}{\left|\eta_{(0)}(x, y)\right|}=(-1)^{k n} *[y] \bullet$ at $\left|T_{(0)}\right|$-a.e. $(x, y)$. The $n$-vector $\xi_{T}$ is called the scaled orientation of $T$. The subscript $T$ in $\xi_{T}$ and in $\tau_{T}$ will often be omitted, for simplicity.

Remark 5.1. If $T=\llbracket G, \eta, \theta \rrbracket \in \operatorname{curv}_{n}\left(\mathbf{R}_{x}^{N}\right)$, then $\eta$ is obtained by normalizing the scaled orientation of $T$, i.e.,

$$
\eta(x, y)=\frac{\xi(x, y)}{|\xi(x, y)|}
$$

at $\left|T_{(0)}\right|$-a.e. $(x, y)$. Moreover, by [11, Theorem 4.3], at $\left|T_{(0)}\right|$-a.e. point $(x, y)$ there exists a class $C^{1}$ embedded submanifold $M$ of $\mathbf{R}_{x}^{N}$, containing $x$ and oriented by a continuous normal simple $k$-vector field $\nu$, approximately differentiable at $x$, such that $\nu(x)=y$ and

$$
\xi(x, y):=\mathbb{A}^{n}(I \oplus \operatorname{ap}-d \nu(x)) \tau(x, y) .
$$

Proposition 5.1. Let $T \in \operatorname{curv}_{\mathrm{n}}\left(\mathbf{R}_{x}^{N}\right)$. Then the following facts are true at $\left|T_{(0)}\right|$-a.e. $(x, y)$ :

1) given $u \in \mathbf{R}_{x}^{N}$, the map

$$
w \mapsto \tilde{A}_{T,(x, y)}^{w}(u)
$$

defines a linear operator in $\mathbf{R}_{x}^{N}$;

2) for all $w \in E_{y}$, one has

$$
\tilde{A}_{T,(x, y)}^{w}\left(E_{\tau(x, y)}\right) \subset E_{\tau(x, y)}
$$

and the restricted operator

$$
A_{T,(x, y)}^{w}:=\left.\tilde{A}_{T,(x, y)}^{w}\right|_{E_{\tau(x, y)}}
$$

is self-adjoint, whereby its eigenvalues are all real numbers. 
In particular, the operator $A_{T,(x, y)}^{w}$ (where $w \in E_{y}$ ) is self-adjoint almost everywhere with respect to $|T|$, provided that $T$ is special.

Proof. (1) This statement is obvious, by (5.1).

(2) Let us recall Remark 5.1. It follows that there exists a class $C^{2}$ embedded submanifold $M^{\prime}$ of $\mathbf{R}_{x}^{N}$, containing $x$ and oriented by a $C^{1}$ normal simple $k$-vector field $\nu^{\prime}$ such that

$$
\nu^{\prime}(x)=\nu(x)=y \quad \text { and } \quad d \nu^{\prime}(x)=\operatorname{ap}-d \nu(x)
$$

which implies

$$
\frac{\eta(x, y)}{\left|\eta_{(0)}(x, y)\right|}=\xi(x, y)=\mathbb{A}^{n}\left(I \oplus d \nu^{\prime}(x)\right) \tau(x, y)
$$

by (5.2). It follows that

$$
\begin{aligned}
A_{M^{\prime}, x}^{w}(v) & =(-1)^{k+n+1}\left[\frac{\eta_{(1)}(x, y)}{\left|\eta_{(0)}(x, y)\right|} \llbracket(\tau(x, y)\llcorner v)] \bullet([\nu(x)] \bullet\llcorner w)\right. \\
& =\tilde{A}_{T,(x, y)}^{w}(v)
\end{aligned}
$$

for all $v \in M_{x}^{\prime}$, by Proposition 3.1 and (5.1). We get the conclusion by noting that $E_{\tau(x, y)}=M_{x}^{\prime}$.

Definition 5.2. The self-adjoint operator

$$
A_{T,(x, y)}^{w}: E_{\tau(x, y)} \rightarrow E_{\tau(x, y)}
$$

of Proposition 5.1(2) will be called the generalized Weingarten operator of $T$ at $(x, y)$ with respect to $w$. Its eigenvalues and eigenvectors will be referred to as generalized principal curvatures and generalized principal directions (with respect to $w$ ), respectively. The corresponding notations will be

$$
\kappa_{j}^{T,(x, y)}(w) \quad \text { and } \quad \tau_{j}^{T,(x, y)}(w)
$$

$j=1, \ldots, n$, but the superscripts will often be omitted to simplify the formulas, whenever it will cause no confusion.

5.1. Generalized second fundamental form and Riemann curvature tensor. Through the classical relations existing among the Weingarten operator, the second fundamental form and the Riemann curvature tensor (e.g., see [9] and [17]), now we can complete the extension to generalized Gauss graphs of the classical curvature machinery. More precisely, if $M$ is a smooth submanifold of $\mathbf{R}_{x}^{N}, p \in M$ and $\left\{w_{j}\right\}$ is an orthonormal basis of $M_{p}^{\perp}$, recall that:

- the second fundamental form of $M$ at $p$

$$
B_{p}: M_{p} \times M_{p} \rightarrow M_{p}^{\perp}
$$


is given by

$$
B_{p}(u, v):=\sum_{j}\left\langle A_{p}^{w_{j}} u \mid v\right\rangle w_{j}
$$

- the Riemann tensor of $M$ at $p$

$$
R_{p}: M_{p} \times M_{p} \times M_{p} \rightarrow M_{p}
$$

satisfies

$$
\begin{aligned}
R_{p}(u, v, z) & :=A_{p}^{B_{p}(u, z)} v-A_{p}^{B_{p}(v, z)} u \\
& =\sum_{j}\left\langle A_{p}^{w_{j}} u \mid z\right\rangle A_{p}^{w_{j}} v-\sum_{j}\left\langle A_{p}^{w_{j}} v \mid z\right\rangle A_{p}^{w_{j}} u .
\end{aligned}
$$

Then, by considering a generalized Gauss graph $T$ and adopting the same notation as in Proposition 5.1, the following definitions become natural.

Definition 5.3. The generalized second fundamental form of $T$

$$
B_{T,(x, y)}: E_{\tau(x, y)} \times E_{\tau(x, y)} \rightarrow E_{\tau(x, y)}^{\perp}=E_{y}
$$

is defined at $\left|T_{(0)}\right|$-a.e. $(x, y)$ as follows:

$$
B_{T,(x, y)}(u, v):=\sum_{j}\left\langle A_{T,(x, y)}^{w_{j}} u \mid v\right\rangle w_{j}
$$

where $\left\{w_{j}\right\}$ is an orthonormal basis of $E_{y}$.

Definition 5.4. The generalized Riemann curvature tensor of $T$

$$
R_{T,(x, y)}: E_{\tau(x, y)} \times E_{\tau(x, y)} \times E_{\tau(x, y)} \rightarrow E_{\tau(x, y)}
$$

is defined at $\left|T_{(0)}\right|$-a.e. $(x, y)$ by:

$$
R_{T,(x, y)}(u, v, z):=\sum_{j}\left\langle A_{T,(x, y)}^{w_{j}} u \mid z\right\rangle A_{T,(x, y)}^{w_{j}} v-\sum_{j}\left\langle A_{T,(x, y)}^{w_{j}} v \mid z\right\rangle A_{T,(x, y)}^{w_{j}} u
$$

where $\left\{w_{j}\right\}$ is an orthonormal basis of $E_{y}$.

In the following proposition, we prove that the generalized second fundamental form and the generalized Riemann curvature tensor are well defined. We obtain also some generalized identities which are well known in the classical case and that could become useful to obtain results concerning our generalized objects from classical arguments.

Proposition 5.2. Definitions 5.3 and 5.4 do not depend on the choice of $\left\{w_{j}\right\}$. Moreover, at $\left|T_{(0)}\right|-$ a.e. $(x, y)$, the following identities hold for all $t, u, v, z \in E_{\tau(x, y)}$ :

1) $B(u, v)=B(v, u)$

2) $R(u, v, z)=A^{B(u, z)} v-A^{B(v, z)} u$

3) $\langle R(u, v, z) \mid t\rangle=-\langle R(u, v, t) \mid z\rangle$

4) $\langle R(u, v, z) \mid t\rangle=-\langle R(v, u, z) \mid t\rangle$ 
5) $\langle R(u, v, z) \mid t\rangle=\langle R(z, t, u) \mid v\rangle$

6) $R(u, v, z)+R(v, z, u)+R(z, u, v)=0$

where we have omitted the subscripts, for the sake of simplicity.

Proof. Let us recall the following simple fact, from the proof of Proposition 5.1: At $\left|T_{(0)}\right|$-a.e. $(x, y)$ there exists a class $C^{2}$ embedded submanifold $M^{\prime}$ of $\mathbf{R}_{x}^{N}$, containing $x$ and oriented by a $C^{1}$ normal simple $k$-vector field $\nu^{\prime}$ such that

$$
E_{y}=E_{\nu^{\prime}(x)}, \quad E_{\tau(x, y)}=M_{x}^{\prime} \quad \text { and } \quad A_{T,(x, y)}^{w}=A_{M^{\prime}, x}^{w}
$$

for all $w \in E_{y}=E_{\nu^{\prime}(x)}$. Therefore, at $\left|T_{(0)}\right|$-a.e. $(x, y)$, the generalized second fundamental form of $T$ at $(x, y)$ coincides with the second fundamental form of $M^{\prime}$ at $x$, whereby it has to be independent from the choice of $\left\{w_{j}\right\}$. The same argument proves that the generalized Riemann curvature tensor is well-defined.

Finally, all the identities follow immediately from Definition 5.3, Definition 5.4 and Proposition 5.1, through a standard computation.

Proposition 5.3. Given $T \in \operatorname{curv}_{\mathrm{n}}\left(\mathbf{R}_{x}^{N}\right)$, the following assertions are true almost everywhere w.r.t. $\left|T_{(0)}\right|$ :

1) if $k=1$ then $|R|=2\left|\xi_{(2)}\right|$

2) if $n=1$ then $|R|=\left|\xi_{(2)}\right|=0$.

In general, one has $|R| \neq\left|\xi_{(2)}\right|$ and there is no constant c such that

$$
\left|\xi_{(2)}\right| \leq c|R|
$$

holds almost everywhere w.r.t. $\left|T_{(0)}\right|$.

Proof. (1) Let $\tau_{j}=\tau_{j}^{T,(x, y)}(y)$ and $\kappa_{j}=\kappa_{j}^{T,(x, y)}(y)$ be the generalized principal directions and curvatures, according to Definition 5.2. Then we get

$$
\left|\xi_{(2)}\right|^{2}=\sum_{i<j} \kappa_{i}^{2} \kappa_{j}^{2}
$$

by Proposition 6.1 below. On the other hand, we have

$$
\begin{aligned}
R_{i j h k}:=\left\langle R\left(\tau_{i}, \tau_{j}\right) \tau_{h} \mid \tau_{k}\right\rangle & =\left\langle A \tau_{i} \mid \tau_{h}\right\rangle\left\langle A \tau_{j} \mid \tau_{k}\right\rangle-\left\langle A \tau_{j} \mid \tau_{h}\right\rangle\left\langle A \tau_{i} \mid \tau_{k}\right\rangle \\
& =\kappa_{i} \kappa_{j}\left(\delta_{i h} \delta_{j k}-\delta_{j h} \delta_{i k}\right)
\end{aligned}
$$

whence, recalling also (5.5), it follows that

$$
\begin{aligned}
|\mathrm{R}|^{2} & =\sum_{i, j, h, k} \mathrm{R}_{i j h k}^{2}=2\left(\sum_{i, j, h, k} \kappa_{i}^{2} \kappa_{j}^{2} \delta_{i h} \delta_{j k}-\sum_{i, j, h, k} \kappa_{i}^{2} \kappa_{j}^{2} \delta_{i h} \delta_{j k} \delta_{j h} \delta_{i k}\right) \\
& =2\left(\sum_{i, j} \kappa_{i}^{2} \kappa_{j}^{2}-\sum_{i} \kappa_{i}^{4}\right)=4 \sum_{i<j} \kappa_{i}^{2} \kappa_{j}^{2}=4\left|\xi_{(2)}\right|^{2} .
\end{aligned}
$$


(2) This statement is trivial.

To verify the last assertion, we will produce a counter-example where $R \equiv 0$ while $\xi_{(2)} \not \equiv 0$. Consider the 2-dimensional submanifold of $\mathbf{R}^{4}$ parametrized by

$$
\varphi\left(x_{1}, x_{2}\right):=\left(x_{1}, x_{2}, x_{1}^{2}, x_{2}^{2}\right): \mathbf{R}^{2} \rightarrow \mathbf{R}^{4}
$$

where we are assuming that $\mathbf{R}^{2}$ and $\mathbf{R}^{4}$ have been equipped with the canonical bases. Then a short calculation shows that the metric matrix and its inverse are given by

$$
\left(g_{i j}\right)=\left(\begin{array}{cc}
1+4 x_{1}^{2} & 0 \\
0 & 1+4 x_{2}^{2}
\end{array}\right) \quad \text { and } \quad\left(g^{i j}\right)=\left(\begin{array}{cc}
\frac{1}{1+4 x_{1}^{2}} & 0 \\
0 & \frac{1}{1+4 x_{2}^{2}}
\end{array}\right) .
$$

By the classical expressions for the Christoffel symbols (e.g., [17], formula (10) on p. 56), we get

$$
\Gamma_{i j}^{m}= \begin{cases}\frac{4 x_{1}}{1+4 x_{1}^{2}} & \text { if } i=j=m=1 \\ \frac{4 x_{2}}{1+4 x_{2}^{2}} & \text { if } i=j=m=2 \\ 0 & \text { otherwise }\end{cases}
$$

and hence it follows immediately (see [17], formula (2) on p. 93) that

$$
R \equiv 0 \text {. }
$$

Now consider the Gauss map

$$
\nu:=\left[\frac{*\left(\frac{\partial \varphi}{\partial x_{1}} \wedge \frac{\partial \varphi}{\partial x_{2}}\right)}{\left|\frac{\partial \varphi}{\partial x_{1}} \wedge \frac{\partial \varphi}{\partial x_{2}}\right|} \circ \varphi^{-1}\right]^{\bullet}
$$

and denote by $T$ the corresponding generalized Gauss graph. The graph of $\nu$ is parametrized by

$$
\Lambda:=(\varphi, \nu \circ \varphi)
$$

i.e.,

$$
\begin{aligned}
\Lambda(x)=\left(x_{1} e_{1}+x_{2} e_{2}+x_{1}^{2} e_{3}+\right. & x_{2}^{2} e_{4}, \\
& \left.\quad a(x)\left[4 x_{1} x_{2} e_{12}-2 x_{1} e_{14}+2 x_{2} e_{23}+e_{34}\right]^{\bullet}\right)
\end{aligned}
$$

where $\left\{e_{j}\right\}$ is the canonical basis of $\mathbf{R}^{4}$, while

$$
a:=\left|\frac{\partial \varphi}{\partial x_{1}} \wedge \frac{\partial \varphi}{\partial x_{2}}\right|^{-1}=\left(1+4 x_{1}^{2}+4 x_{2}^{2}+16 x_{1}^{2} x_{2}^{2}\right)^{-1 / 2} .
$$

Hence, by a quite boring computation and noting that

$$
a(0)=1 \quad \text { and } \quad \nabla a(0)=0,
$$


we obtain

$$
\frac{\partial \Lambda}{\partial x_{1}}(0) \mathbb{\wedge} \frac{\partial \Lambda}{\partial x_{2}}(0)=\left(e_{1},-2\left[e_{14}\right]^{\bullet}\right) \mathbb{A}\left(e_{2}, 2\left[e_{23}\right]^{\bullet}\right) .
$$

It follows that

$$
\left[\frac{\partial \Lambda}{\partial x_{1}}(0) \mathbb{\wedge} \frac{\partial \Lambda}{\partial x_{2}}(0)\right]_{(2)}=-4\left(0,\left[e_{14}\right]^{\bullet}\right) \mathbb{\wedge}\left(0,\left[e_{23}\right]^{\bullet}\right) \neq 0
$$

whereby the scaled orientation of $T$ has to be different from zero at every point of a neighbourhood of $\Lambda(0)$.

\section{Length of strata of the scaled orientation.}

Consider $T \in \operatorname{curv}_{\mathrm{n}}\left(\mathbf{R}_{x}^{N}\right)$. Then, at $\left|T_{(0)}\right|$ almost all $(x, y)$, given an orthonormal basis $\left\{w_{j}\right\}$ of $E_{y}$, one can find the generalized principal curvatures and directions of $T$ (according to Definition 5.2). We will denote them by $\kappa_{l}\left(w_{j}\right)$ and $\tau_{l}\left(w_{j}\right)$ respectively, omitting mention of the superscripts, for simplicity.

The goal of this section is computing the length of the $h$-th stratum $\xi_{(h)}$ of the scaled orientation $\xi=\xi_{T}$. Consider an orthonormal basis $\left\{u_{j}\right\}$ for the enveloping subspace of $\tau=\tau_{T}$ and then define $J, X_{h}^{*}, X_{h}, \tau_{\gamma}, \kappa_{\gamma}, \tau_{\Gamma}$, $\kappa_{\Gamma}$ as in subsection $\S 3.2$. We have the following result.

Proposition 6.1. For $h=1, \ldots, n$ one has

$$
\left|\xi_{(h)}\right|^{2}=\sum_{\Gamma \in X_{h}} \kappa_{\Gamma}^{2}\left|\tau_{\Gamma}\right|^{2} \quad\left(\text { hence }|\xi|^{2}=1+\sum_{h=1}^{n} \sum_{\Gamma \in X_{h}} \kappa_{\Gamma}^{2}\left|\tau_{\Gamma}\right|^{2}\right)
$$

In particular,

$$
\left|\xi_{(1)}\right|^{2}=\sum_{\Gamma \in X_{1}} \kappa_{\Gamma}^{2}=\sum_{j, l} \kappa_{l}\left(w_{j}\right)^{2}=\sum_{j}\left|A^{w_{j}}\right|^{2} .
$$

Moreover, the following estimate holds whenever $1 \leq l \leq h$ :

$$
\left|\xi_{(h)}\right| \leq c(n, k)\left|\xi_{(l)}\right|^{h / l} .
$$

Proof. Recalling Equation (5.3) we get:

$$
\begin{aligned}
\xi(x, y) & =\mathbb{A}^{n}\left(I \oplus d \nu_{x}^{\prime}\right)\left(u_{1} \wedge \cdots \wedge u_{n}\right)=\left(u_{1}, d \nu_{x}^{\prime}\left(u_{1}\right)\right) \mathbb{A} \cdots \mathbb{A}\left(u_{n}, d \nu_{x}^{\prime}\left(u_{n}\right)\right) \\
& =\tau(x, y)+\sum_{h=1}^{n} \sum_{\alpha \in I(n, h)} \sigma(\alpha, \bar{\alpha})\left[\left(\mathbb{A}^{h} d \nu_{x}^{\prime}\right) u_{\alpha}\right] \mathbb{A} u_{\bar{\alpha}} .
\end{aligned}
$$

It follows (by dropping the obvious notation)

$$
\xi_{(h)}=\sum_{\alpha \in I(n, h)} \sigma(\alpha, \bar{\alpha})\left[\left(\mathbb{\aleph}^{h} d \nu^{\prime}\right) u_{\alpha}\right] \mathbb{\wedge} u_{\bar{\alpha}}
$$


for $h=1, \ldots, n$, whence

$$
\left|\xi_{(h)}\right|^{2}=\sum_{\alpha \in I(n, h)}\left|\left(\mathbb{A}^{h} d \nu^{\prime}\right) u_{\alpha}\right|^{2}
$$

for $h=1, \ldots, n$.

By identities (3.2) and (5.4), we find

$$
\left[d \nu_{x}^{\prime}(v)\right]_{\bullet}=\sum_{j}(-1)^{j} A_{T,(x, y)}^{w_{j}}(v) \wedge w_{\bar{j}}
$$

for all $v \in E_{\tau(x, y)}$ and then also

$$
\begin{aligned}
\left(\mathbb{A}^{h} d \nu^{\prime}\right) u_{\alpha} & \\
= & \sum_{j_{1}, \ldots, j_{h}}(-1)^{j_{1}+\cdots+j_{h}}\left[A_{T}^{w_{j_{1}}}\left(u_{\alpha_{1}}\right) \wedge w_{\overline{j_{1}}}\right] \bullet \mathbb{A} \cdots \mathbb{\wedge}\left[A_{T}^{w_{j_{h}}}\left(u_{\alpha_{h}}\right) \wedge w_{\overline{j_{h}}}\right] \\
= & \sum_{\substack{j_{1}, \ldots, j_{h} \\
l_{1}, \ldots, l_{h}}}^{\bullet}(-1)^{j_{1}+\cdots+j_{h}}\left\langle u_{\alpha_{1}} \mid \tau_{l_{1}}\left(w_{j_{1}}\right)\right\rangle \cdots\left\langle u_{\alpha_{h}} \mid \tau_{l_{h}}\left(w_{j_{h}}\right)\right\rangle \cdot \\
& \cdot \kappa_{l_{1}}\left(w_{j_{1}}\right) \cdots \kappa_{l_{h}}\left(w_{j_{h}}\right)\left[\tau_{l_{1}}\left(w_{j_{1}}\right) \wedge w_{\overline{j_{1}}}\right] \bullet \mathbb{M} \cdots \mathbb{A}\left[\tau_{l_{h}}\left(w_{j_{h}}\right) \wedge w_{\overline{j_{h}}}\right] .
\end{aligned}
$$

Recalling the notation introduced above and (3.4), we obtain

$$
\begin{aligned}
& \left(\mathbb{A}^{h} d \nu^{\prime}\right) u_{\alpha} \\
& =\sum_{\substack{\Gamma \in X_{h}^{*} \\
\Gamma=\left(\gamma_{1}, \cdots, \gamma_{h}\right)}}(-1)^{j_{\gamma_{1}}+\cdots+j_{\gamma_{h}}}\left\langle u_{\alpha_{1}} \mid \tau_{\gamma_{1}}\right\rangle \cdots\left\langle u_{\alpha_{h}} \mid \tau_{\gamma_{h}}\right\rangle . \\
& \quad \cdot \kappa_{\Gamma}\left[\tau_{\gamma_{1}} \wedge w_{\overline{j_{\gamma_{1}}}}\right]^{\bullet} \mathbb{\wedge} \cdots \mathbb{\wedge}\left[\tau_{\gamma_{h}} \wedge w_{\overline{j_{\gamma_{h}}}}\right] \\
& \left.=\sum_{\substack{\Gamma \in X_{h} \\
\Gamma=\left(\gamma_{1}, \cdots, \gamma_{h}\right)}}(-1)^{j_{\gamma_{1}}+\cdots+j_{\gamma_{h}}\left\langle\tau_{\Gamma} \mid u_{\alpha}\right\rangle \kappa_{\Gamma}\left[\tau_{\gamma_{1}} \wedge w_{\overline{j_{1}}}\right]}\right]^{\bullet} \mathbb{A} \cdots \mathbb{A}\left[\tau_{\gamma_{h}} \wedge w_{\overline{j_{\gamma_{h}}}}\right] .
\end{aligned}
$$

From the identity

$$
\left\langle\left[\tau_{l}\left(w_{j}\right) \wedge w_{\bar{j}}\right]^{\bullet} \mid\left[\tau_{l^{\prime}}\left(w_{j^{\prime}}\right) \wedge w_{\overline{j^{\prime}}}\right]^{\bullet}\right\rangle=\delta_{l l^{\prime}} \delta_{j j^{\prime}}
$$

together with (6.1), we get

$$
\left|\xi_{(h)}\right|^{2}=\sum_{\Gamma \in X_{h}} \kappa_{\Gamma}^{2} \sum_{\alpha \in I(n, h)}\left\langle\tau_{\Gamma} \mid u_{\alpha}\right\rangle^{2}=\sum_{\Gamma \in X_{h}} \kappa_{\Gamma}^{2}\left|\tau_{\Gamma}\right|^{2}
$$

that is just the first equality we had to prove. Now the second one is clear, while the inequality follows from Proposition 6.2. 
Proposition 6.2. If $l$ and $h$ are integers satisfying $1 \leq l \leq h$, then there exists a positive constant $c(h, l)$ such that

$$
\left|v_{1} \wedge \cdots \wedge v_{h}\right| \leq c(l, h) \sum_{\beta \in I(h, l)}\left|v_{\beta_{1}} \wedge \cdots \wedge v_{\beta_{l}}\right|^{\frac{h}{l}}
$$

for every set of vectors $\left\{v_{1}, \ldots, v_{h}\right\} \subset \mathbf{R}^{n}$.

Proof. Let us indicate with $G S\left\{z_{1}, \ldots, z_{m}\right\}$ the orthogonal set $\left\{z_{1}^{\prime}, \ldots, z_{m}^{\prime}\right\}$ obtained by processing a linearly independent set $\left\{z_{1}, \ldots, z_{m}\right\} \subset \mathbf{R}^{n}$, through the well-known Gram-Schmidt orthogonalization algorithm:

$$
z_{1}^{\prime}:=z_{1}, \quad z_{2}^{\prime}:=z_{2}-\left\langle z_{2} \mid \widehat{z_{1}^{\prime}}\right\rangle \widehat{z_{1}^{\prime}}, \quad z_{3}^{\prime}:=z_{3}-\left\langle z_{3} \mid \widehat{z_{1}^{\prime}}\right\rangle \widehat{z_{1}^{\prime}}-\left\langle z_{3} \mid \widehat{z_{2}^{\prime}}\right\rangle \widehat{z_{2}^{\prime}}
$$

et cetera, where $\hat{z}$ denotes the unit vector $z /|z|$. Obviously one has

$$
\left|z_{1} \wedge \cdots \wedge z_{m}\right|=\left|z_{1}^{\prime} \wedge \cdots \wedge z_{m}^{\prime}\right|=\left|z_{1}^{\prime}\right| \cdots\left|z_{m}^{\prime}\right| .
$$

Then let $v_{1}, \ldots, v_{h}$ be linearly independent (otherwise (6.2) trivially holds for all nonnegative constants $c$ ) and consider

$$
\left\{v_{1}^{\prime}, \ldots, v_{h}^{\prime}\right\}:=G S\left\{v_{1}, \ldots, v_{h}\right\}, \quad\left\{v_{\beta_{1}}^{\prime \prime}, \ldots, v_{\beta_{l}}^{\prime \prime}\right\}:=G S\left\{v_{\beta_{1}}, \ldots, v_{\beta_{l}}\right\}
$$

where $\beta \in I(h, l)$. From (6.3), (6.4) which will be proved below, and since

$$
\left|v_{\beta_{j}}^{\prime}\right| \leq\left|v_{\beta_{j}}^{\prime \prime}\right|
$$

we get

$$
\begin{aligned}
\left|v_{1} \wedge \cdots \wedge v_{h}\right| & =\left|v_{1}^{\prime}\right| \cdots\left|v_{h}^{\prime}\right| \leq c(l, h) \sum_{\beta \in I(h, l)}\left(\left|v_{\beta_{1}}^{\prime}\right| \cdots\left|v_{\beta_{l}}^{\prime}\right|\right)^{\frac{h}{l}} \\
& \leq c(l, h) \sum_{\beta \in I(h, l)}\left(\left|v_{\beta_{1}}^{\prime \prime}\right| \cdots\left|v_{\beta_{l}}^{\prime \prime}\right|\right)^{\frac{h}{l}} \\
& =c(l, h) \sum_{\beta \in I(h, l)}\left|v_{\beta_{1}} \wedge \cdots \wedge v_{\beta_{l}}\right|^{\frac{h}{l}}
\end{aligned}
$$

i.e., just (6.2).

It remains to prove the following assertion which has been used above: There exists a positive constant $c(h, l)$ such that

$$
x_{1} \cdots x_{h} \leq c(l, h) \sum_{\beta \in I(h, l)}\left(x_{\beta_{1}} \cdots x_{\beta_{l}}\right)^{\frac{h}{l}}
$$

for every set of nonnegative real numbers $\left\{x_{1}, \ldots, x_{h}\right\}$.

Let us set $x:=\left(x_{1}, \ldots, x_{h}\right)$ and note that, by homogeneity, one can assume also $|x|=1$. We shall prove by induction on $l$ that

$$
\text { the statement holds for all } h \geq l \text {. }
$$


Let $l=1, h \geq l$ and $m:=\max _{j} x_{j}$. Then the assertion (6.5) has to be true in that

$$
\sum_{j} x_{j}^{\frac{h}{1}} \geq m^{h} \geq x_{1} \cdots x_{h} .
$$

Now, assume assertion (6.5) be true and prove the inductive step. Let $h \geq l+1$ and consider

$$
f(x):=\sum_{\beta \in I(h, l+1)}\left(x_{\beta_{1}} \cdots x_{\beta_{l+1}}\right)^{\frac{h}{l+1}} .
$$

We get

$$
\begin{aligned}
f(x) & \geq x_{h}^{\frac{h}{l+1}} \sum_{\beta \in I(h-1, l)}\left(x_{\beta_{1}} \cdots x_{\beta_{l}}\right)^{\frac{h}{l+1}} \\
& \geq c(h, l) x_{h}^{\frac{h}{l+1}}\left[\sum_{\beta \in I(h-1, l)}\left(x_{\beta_{1}} \cdots x_{\beta_{l}}\right)^{\frac{h-1}{l}}\right]^{\frac{h l}{(h-1)(l+1)}} \\
& \geq c(h, l) x_{h}^{\frac{h}{l+1}}\left(x_{1} \cdots x_{h-1}\right)^{\frac{h l}{(h-1)(l+1)}} \\
& =c(h, l) x_{h}^{\frac{h}{l+1}-\frac{h l}{(h-1)(l+1)}}\left(x_{1} \cdots x_{h}\right)^{\frac{h l}{(h-1)(l+1)}}
\end{aligned}
$$

Since

$$
x_{1} \cdots x_{h} \leq 1 \quad \text { and } \quad \frac{h l}{(h-1)(l+1)} \leq 1
$$

we find

$$
f(x) \geq c(h, l) x_{h}^{\rho(h, l)} x_{1} \cdots x_{h}
$$

where

$$
\rho(h, l):=\frac{h}{l+1}-\frac{h l}{(h-1)(l+1)} .
$$

It follows that

$$
f(x) \geq c(h, l) x_{j}^{\rho(h, l)} x_{1} \cdots x_{h}
$$

for all $j$, and hence also

$$
f(x) \geq c(h, l)\left(\max _{j} x_{j}\right)^{\rho(h, l)} x_{1} \cdots x_{h} .
$$

We obtain the conclusion by recalling that $x \mapsto \max _{j}\left|x_{j}\right|$ defines a norm in $\mathbf{R}^{h}$ and that the norms in $\mathbf{R}^{h}$ are all equivalent.

Remark 6.1. Proposition 6.1 shows, in particular, that the expression

$$
L_{T}:=\sqrt{\sum_{j, l} \kappa_{l}\left(w_{j}\right)^{2}}
$$

does not depend on the choice of the basis $\left\{w_{j}\right\}$. This result generalizes a fact which is well known in the classical case of a smooth immersed submanifold 
of $\mathbf{R}^{N}$. Then, by extending the classical terminology, $L_{T}$ will be referred to as the length of the second fundamental form of $T$ (compare [6, §29.4.2]).

Remark 6.2. The estimate stated in Proposition 6.1 generalizes the one we got in the proof of the smooth estimate [14, Theorem 5.2].

\section{Integrals of generalized curvatures: Some results in view of variational applications.}

The first proposition we state in this section, is a semicontinuity result (in the special case $k=1$ ), which can be used to solve geometric variational problems by the direct method. Then we will get Proposition 7.2 and its corollary Proposition 7.3, which make possible to define integral functionals of symmetric functions of generalized curvatures, e.g., mean curvature or Lipschitz-Killing curvature.

Finally, we prove some integral estimates which allow to get compactness results for problems involving special generalized Gauss graphs (see [13]). Applications will be given in the last section.

7.1. A semicontinuity result. Let us introduce some notation. If $T \in$ $\operatorname{curv}_{n}^{*}\left(\mathbf{R}_{x}^{n+1}\right)$ then, at $\left|T_{(0)}\right|$ almost every $(x, y)$, we set:

$$
\gamma_{T}(x, y):=\max _{j}\left(\kappa_{j}^{T,(x, y)}(y) \bigvee 0\right)=\max _{\substack{u \in E_{\tau(x, y)} \\|u| \leq 1}}\left\langle A_{T,(x, y)}^{y}(u) \mid u\right\rangle
$$

and

$$
\lambda_{T}(x, y):=\min _{j}\left(\kappa_{j}^{T,(x, y)}(y) \bigwedge 0\right)=\min _{\substack{u \in E_{\tau(x, y)} \\|u| \leq 1}}\left\langle A_{T,(x, y)}^{y}(u) \mid u\right\rangle .
$$

The maps $\gamma_{T}$ and $\lambda_{T}$ are evidently $\mathcal{H}^{n}$-measurable and have constant sign: $\gamma_{T}$ is nonnegative while $\lambda_{T}$ is nonpositive. Note also that

$$
-\lambda_{T}(x, y)=\max _{\substack{u \in E_{\tau(x, y)} \\|u| \leq 1}}\left\langle-A_{T,(x, y)}^{y}(u) \mid u\right\rangle .
$$

Finally denote the usual operator norm by $|\|\cdot|\||$, so that

$$
\begin{aligned}
\left\|A_{T,(x, y)}^{y} \mid\right\| & =\max _{\substack{u \in E_{\tau(x, y)} \\
|u| \leq 1}}\left|A_{T,(x, y)}^{y}(u)\right| \\
& =\max _{\substack{u \in E_{\tau(x, y)} \\
|u| \leq 1}} \sqrt{\left\langle A_{T,(x, y)}^{y}(u) \mid A_{T,(x, y)}^{y}(u)\right\rangle}=\max _{j}\left|\kappa_{j}^{T,(x, y)}(y)\right| \\
& =\max \left\{\gamma_{T}(x, y),-\lambda_{T}(x, y)\right\}
\end{aligned}
$$

since the Weingarten operator is self-adjoint, by Proposition 5.1.

Now we are ready to state the announced result. 
Proposition 7.1. If $T_{j}, T \in \operatorname{curv}_{n}^{*}\left(\mathbf{R}_{x}^{n+1}\right)$ are such that $T_{j} \rightarrow T$, then the following inequalities hold:

1) $\int \gamma_{T} d\left|T_{(0)}\right| \leq \liminf _{j} \int \gamma_{T_{j}} d\left|T_{j(0)}\right|$

2) $\int \lambda_{T} d\left|T_{(0)}\right| \geq \lim \sup _{j} \int \lambda_{T_{j}} d\left|T_{j_{(0)}}\right|$

3) $\int||\left|A_{T}\right||| d\left|T_{(0)}\right| \leq \liminf _{j} \int||\left|A_{T_{j}}\right||| d\left|T_{j_{(0)}}\right|$

where the notation has been reduced, for the convenience of the reader.

Proof. Let $\llbracket G, \eta, \theta \rrbracket$ and $\llbracket G_{j}, \eta_{j}, \theta_{j} \rrbracket$ be representations of $T$ and $T_{j}$, respectively.

(1) Consider a field

$$
F \in C_{c}^{\infty}\left(\mathbf{R}_{x}^{n+1} \times \mathbf{R}_{y}^{n+1}, \mathbf{R}_{x}^{n+1}\right)
$$

and let $P F$ be its orthogonal projection on $E_{\tau}$, i.e.,

$$
P F(x, y):=F(x, y)-\left\langle F(x, y) \mid[y]_{\bullet}\right\rangle[y]_{\bullet} .
$$

Since $T_{j}$ and $T$ are special generalized Gauss graphs and by definition of Weingarten operator, we get

$$
\begin{aligned}
& \lim _{j} \int\left\langle A_{T_{j}}(P F) \mid P F\right\rangle d\left|T_{j(0)}\right| \\
& =\lim _{j} \int_{G_{j}}\left\langle\eta_{j}\right|\left(*[y] \bullet\llcorner(P F)) \mathbb{A}[P F]^{\bullet}\right\rangle \theta_{j} d \mathcal{H}^{n} \\
& =\int_{G}\langle\eta|\left(*[y] \bullet\llcorner(P F)) \mathbb{A}[P F]^{\bullet}\right\rangle \theta d \mathcal{H}^{n} \\
& =\int\left\langle A_{T}(P F) \mid P F\right\rangle d\left|T_{(0)}\right|
\end{aligned}
$$

and hence

$$
\int\left\langle A_{T}(P F) \mid P F\right\rangle d\left|T_{(0)}\right| \leq \liminf _{j} \int \gamma_{T_{j}} d\left|T_{j_{(0)}}\right|
$$

for all $F$ such that $\sup |F| \leq 1$. The inequality follows by the arbitariness of $F$.

(2) From (7.2) and (7.1) we obtain also

$$
\begin{aligned}
\int\left\langle-A_{T}(P F) \mid P F\right\rangle d\left|T_{(0)}\right| & \leq \liminf _{j} \int-\lambda_{T_{j}} d\left|T_{j(0)}\right| \\
& =-\limsup _{j} \int \lambda_{T_{j}} d\left|T_{j_{(0)}}\right|
\end{aligned}
$$

provided that $\sup |F| \leq 1$, whence we conclude by the arbitrariness of $F$, as before. 
(3) If $F_{1}, F_{2} \in C_{c}^{\infty}\left(\mathbf{R}_{x}^{n+1} \times \mathbf{R}_{y}^{n+1}, \mathbf{R}_{x}^{n+1}\right)$ then, as before, one finds

$$
\begin{aligned}
\int\left\langle A_{T}\left(P F_{1}\right) \mid P F_{2}\right\rangle d\left|T_{(0)}\right| & =\lim _{j} \int\left\langle A_{T_{j}}\left(P F_{1}\right) \mid P F_{2}\right\rangle d\left|T_{j_{(0)}}\right| \\
& \leq \liminf _{j} \int \| A_{T_{j}}|| d\left|T_{j(0)}\right|
\end{aligned}
$$

whenever $\sup \left|F_{i}\right| \leq 1(i=1,2)$. Once again the conclusion comes from the arbitariness of the vector fields.

7.2. Measurability of symmetric functions of generalized curvatures. Given a current

$$
T=\llbracket G, \eta, \theta \rrbracket \in \operatorname{curv}_{\mathrm{n}}\left(\mathbf{R}_{x}^{N}\right),
$$

let $w$ be a $\mathcal{H}^{n}\left\llcorner G^{*}\right.$-measurable map such that $w(x, y) \in E_{y}$ almost everywhere and consider the characteristic polynomial of $A_{T,(x, y)}^{w(x, y)}$ :

$$
p^{(x, y)}(\lambda)=a_{n}(x, y) \lambda^{n}+a_{n-1}(x, y) \lambda^{n-1}+\cdots+a_{0}(x, y) .
$$

Note that the coefficients $a_{j}$ are measurable w.r.t. $\mathcal{H}^{n}\left\llcorner G^{*}\right.$.

Proposition 7.2. There exists a complete system of measurable trajectories of principal curvatures with respect to the map $w$. In other words, there exist measurable maps

$$
\mu_{i}: G^{*} \rightarrow \mathbf{R} \quad(i=1, \ldots, n)
$$

such that

$$
p^{(x, y)}(\lambda)=a_{n}(x, y)\left(\lambda-\mu_{1}(x, y)\right) \cdots\left(\lambda-\mu_{n}(x, y)\right)
$$

at $\mathcal{H}^{n}\left\llcorner G^{*}\right.$-a.e. $(x, y)$.

Proof. It will be enough to prove the existence of one of such measurable trajectories. Then the conclusion will follow from the division rule for polynomials, by induction.

Let $C: G^{*} \rightarrow \mathbf{R}^{n+1}$ and $Z: \mathbf{R}^{n+1} \rightarrow \mathcal{P}(\mathbf{R})$ be a couple of maps defined as follows:

$$
\left\{\begin{array}{l}
C(x, y):=\left(a_{0}(x, y), \ldots, a_{n}(x, y)\right) \\
Z\left(t_{0}, \ldots, t_{n}\right):=\left\{\lambda \in \mathbf{R} \mid \sum_{j=0}^{n} t_{j} \lambda^{j}=0\right\} .
\end{array}\right.
$$

By [8, Corollary III.3, p. 63] we get that $Z$ is measurable w.r.t. $\mathcal{B}\left(\mathbf{R}^{n+1}\right)$. Then $F:=Z \circ C$ is measurable w.r.t. $\mathcal{H}^{n}\left\llcorner G^{*}\right.$ and hence the set

$$
\left\{(x, y) \in G^{*} \mid F(x, y) \cap U \neq \emptyset\right\}
$$

is measurable w.r.t. $\mathcal{H}^{n}\left\llcorner G^{*}\right.$ whenever $U \subset \mathbf{R}$ is open, by [8, Theorem III.2, p. 62]. 
Now, Theorem III.6 of $[8]$, p. 65 , implies that $F$ admits a measurable (w.r.t. $\mathcal{H}^{n}\left\llcorner G^{*}\right.$ ) selection, i.e., a measurable function

$$
\mu: G^{*} \rightarrow \mathbf{R}
$$

such that $\mu(x, y) \in F(x, y)=\left\{\lambda \in \mathbf{R} \mid p^{(x, y)}(\lambda)=0\right\}$.

As a consequence, we get immediately the following corollary. By analogy with $[\mathbf{6}, \S 29.3]$, let us denote by $\sigma_{i}(x, y ; w)$ the $i$-th elementary symmetric function of principal curvatures at the point $(x, y)$ with respect to the normal vector $w$.

Proposition 7.3. Under the same assumptions of Proposition 7.2, the maps

$$
(x, y) \mapsto \sigma_{i}(x, y ; w(x, y))
$$

are measurable with respect to $\mathcal{H}^{n}\left\llcorner G^{*}\right.$.

7.3. Some integral estimates. As an immediate corollary of Proposition 6.1 and Hölder inequality, we get:

Proposition 7.4. If $q$ is a nonnegative real number and $l, h$ are integers such that $1 \leq l \leq h \leq n$, then

$$
E_{q}^{(h)}(T) \leq c(n, k, q) E_{\frac{q h}{l}}^{(l)}(T)
$$

for all $T \in \operatorname{curv}_{\mathrm{n}}\left(\mathbf{R}_{x}^{N}\right)$. Moreover, if $p$ is a positive real number such that $p \geq q$ and if $h \in\{0,1, \ldots, n\}$, then one has

$$
E_{q}^{(h)}(T) \leq E_{p}^{(h)}(T)^{\frac{q}{p}}\left\|T_{(0)}\right\|^{1-\frac{q}{p}}
$$

for all $T \in \operatorname{curv}_{\mathrm{n}}\left(\mathbf{R}_{x}^{N}\right)$.

Hence the following result follows.

Proposition 7.5. Let $p$ be a positive real number, $l \in\{1,2, \ldots, n\}$ and $q \in\left[0, \frac{l p}{n}\right]$. Then

$$
\begin{aligned}
\mathcal{G}_{F_{q}}(T) \leq\left\|T_{(0)}\right\|+E_{q}^{(1)}(T)+\cdots+ & E_{q}^{(l-1)}(T) \\
& +c(n, k, q) \sum_{h=l}^{n} E_{p}^{(l)}(T)^{\frac{q h}{p l}}\left\|T_{(0)}\right\|^{1-\frac{q h}{p l}}
\end{aligned}
$$

for all $T \in \operatorname{curv}_{\mathrm{n}}\left(\mathbf{R}_{x}^{N}\right)$.

In particular, one has

$$
\begin{aligned}
\mathbf{M}(T) \leq\left\|T_{(0)}\right\|+E_{1}^{(1)}(T)+\cdots+E_{1}^{(l-1)} & (T) \\
& +c(n, k) \sum_{h=l}^{n} E_{p}^{(l)}(T)^{\frac{h}{p l}}\left\|T_{(0)}\right\|^{1-\frac{h}{p l}}
\end{aligned}
$$

for all $T \in \operatorname{curv}_{\mathrm{n}}^{*}\left(\mathbf{R}_{x}^{N}\right)$, provided that $l p \geq n$. 
Proof. Let us recall from the subsection $\S 4.2$ that

$$
E_{q}^{(0)}(T)=\left\|T_{(0)}\right\| \quad \text { and } \quad \mathcal{G}_{F_{q}}(T)=\sum_{h=0}^{n} E_{q}^{(h)}(T) .
$$

Then the inequality follows at once by Proposition 7.4.

Remark 7.1. Let $V$ be a $n$-dimensional, compact and orientable Riemannian manifold without boundary and consider the set

$$
\operatorname{Imm}^{2, p}\left(V, \mathbf{R}_{x}^{n+1}\right):=\left\{\varphi \in H^{2, p}\left(V, \mathbf{R}_{x}^{n+1}\right) \mid \varphi \text { is an immersion }\right\}
$$

where $p>n$. Let us recall that the area of $\varphi \in \operatorname{Imm}^{2, p}\left(V, \mathbf{R}_{x}^{n+1}\right)$ is defined in a natural way by

$$
\operatorname{Area}(\varphi):=\int_{V}|d \varphi(\tau)|
$$

where $\tau$ denotes a smooth orientation of $V$. In [14] we showed that, given $\varphi \in \operatorname{Imm}^{2, p}\left(V, \mathbf{R}_{x}^{n+1}\right)$, the corresponding generalized Gauss graph $T_{\varphi}$ is special and has null boundary, i.e.,

$$
T_{\varphi} \in \operatorname{curv}_{\mathrm{n}}^{*}\left(\mathbf{R}_{x}^{n+1}\right) \quad \text { and } \quad \partial T_{\varphi}=0 .
$$

Note also that

$$
\operatorname{Area}(\varphi)=\left\|T_{\varphi(0)}\right\|
$$

Then, by Proposition 7.5, one gets immediately the following estimate. In the special case $l=1$, we obtain a result which was conjectured to be true in $[\mathbf{1 4}]$ (where it was proved for $\varphi$ of class $C^{2}$ ).

Proposition 7.6. Given a positive real number $p$ and $l \in\{1,2, \ldots, n\}$, the estimate

$$
\begin{aligned}
\mathcal{G}_{F_{q}}\left(T_{\varphi}\right) \leq \operatorname{Area}(\varphi)+E_{q}^{(1)}\left(T_{\varphi}\right)+ & \cdots+E_{q}^{(l-1)}\left(T_{\varphi}\right)+ \\
& +c(n, k, q) \sum_{h=l}^{n} E_{p}^{(l)}\left(T_{\varphi}\right)^{\frac{q h}{p l}} \operatorname{Area}(\varphi)^{1-\frac{q h}{p l}}
\end{aligned}
$$

holds for all $\varphi \in \operatorname{Imm}^{2, p}\left(V, \mathbf{R}_{x}^{n+1}\right)$ and $q \in\left[0, \frac{l p}{n}\right]$.

\section{Variational applications.}

In this section we give some simple examples where the results stated above are used to handle variational problems. 
8.1. First application. Consider the problem of minimizing the $L^{r}$ norm of the Riemann curvature tensor, for $r>n / 2$, among the immersions $\varphi \in$ $\operatorname{Imm}^{2, p}\left(V, \mathbf{R}_{x}^{n+1}\right)$ (see Remark 7.1) such that

(i) $\operatorname{Area}(\varphi) \leq A, \quad|\operatorname{Bar}(\varphi)| \leq B \quad$ and $\quad E_{p}^{(1)}\left(T_{\varphi}\right) \leq C$

(ii) $\varphi(V) \supset M$

where $A, B, C$ are fixed positive constants, $\operatorname{Bar}(\varphi)$ is the "center of gravity" of $\varphi$ (see [14]), and $M$ is a given $n$-dimensional rectifiable subset of $\mathbf{R}_{x}^{n+1}$. Denote by $\mathcal{D}$ the set of such immersions, that is

$$
\mathcal{D}:=\left\{\varphi \in \operatorname{Imm}^{2, p}\left(V, \mathbf{R}_{x}^{n+1}\right) \mid \text { (i) and (ii) are satisfied }\right\}
$$

with $p>n$.

Proposition 8.1. Let $\mathcal{F}$ be the functional defined in $\mathcal{D}$ as follows

$$
\mathcal{F}(\varphi):=2^{r} E_{r}^{(2)}\left(T_{\varphi}\right)
$$

i.e., $\mathcal{F}$ is the $r$-th power of the $L^{r}$ norm of the Riemann curvature tensor, by Proposition 5.3. Then $\mathcal{F}$ has a minimizer, provided that $r>n / 2$.

Proof. Assume

$$
\inf \mathcal{F}=m<+\infty
$$

otherwise the assertion is trivial, and let $\varphi_{j}$ be a minimizing sequence. By [14, Theorem 7.1] and [12, Proposition 5.1], there exist a subsequence $\varphi_{j^{\prime}}$ and diffeomorphisms $\Delta_{j^{\prime}}: V \rightarrow V$ such that the $\varphi_{j^{\prime}} \circ \Delta_{j^{\prime}}$ converge in the $\mathcal{H}^{2, p}$ weak topology to an immersion $\varphi \in \mathcal{D}$. It follows that

$$
T_{\varphi_{j^{\prime}}}=T_{\varphi_{j^{\prime}} \circ \Delta_{j^{\prime}}} \rightarrow T_{\varphi}
$$

for $[\mathbf{1 4}$, Corollary 5.6]. Hence, applying [14, Theorem 4.2] with $F(\xi)=$ $\left|\xi_{(2)}\right|^{r}$, we get

$$
m \leq \mathcal{F}\left(T_{\varphi}\right) \leq \liminf _{j^{\prime}} \mathcal{F}\left(T_{\varphi_{j^{\prime}}}\right)=m
$$

i.e., $\varphi$ is a minimizer.

Remark 8.1. Let $\left\{T_{j}\right\} \subset \operatorname{curv}_{\mathrm{n}}^{*}\left(\mathbf{R}_{x}^{n+1}\right)$ be such that

$$
\sup _{j}\left\{\int\left(1+L_{T_{j}}+\left|R_{T_{j}}\right|^{\frac{n}{2}}\right) d\left|T_{j(0)}\right|\right\}<+\infty
$$

where $L_{T_{j}}$ denotes the length of the second fundamental form of $T_{j}$, according to Remark 6.1. By Proposition 7.5 and Proposition 5.3, the masses $\mathbf{M}\left(T_{j}\right)$ must be equi-bounded. If $\mathbf{M}\left(\partial T_{j}\right)$ are also uniformly bounded, then there exists a subsequence $\left\{T_{j^{\prime}}\right\}$ which converges weakly to $T \in \operatorname{curv}_{\mathrm{n}}\left(\mathbf{R}_{x}^{n+1}\right)$. It is easy to find situations (e.g., shrinking spherical surfaces in $\mathbf{R}^{3}$ ) showing that condition (8.1) does not prevent the limit Gauss graph from having vertical parts of positive measure. In other words, under the assumption 
(8.1), it can happen that $T$ is not a special generalized Gauss graph. To be sure that $T \in \operatorname{curv}_{\mathrm{n}}^{*}\left(\mathbf{R}_{x}^{n+1}\right)$, it is enough to replace (8.1) by

$$
\sup _{j}\left\{\int\left(1+L_{T_{j}}^{q}+\left|R_{T_{j}}\right|^{p}\right) d\left|T_{j_{(0)}}\right|\right\}<+\infty
$$

where $p>n / 2$ and $1<q \leq 2 p / n$. Indeed, by Proposition 7.5 and Proposition 5.3, we can apply the compactness theorem [13, Corollary 4.2].

8.2. Second application. The domain of the functional will be the set $\mathcal{D}$ of currents

$$
T=\llbracket G, \eta, \theta \rrbracket \in \operatorname{curv}_{\mathrm{n}}^{*}\left(\mathbf{R}_{x}^{N}\right) \quad(N=n+k)
$$

such that

(i) $\left\|T_{(0)}\right\| \leq A, \quad E_{q}^{(i)}(T) \leq B(i=1, \ldots, l-1), \quad$ and $\quad E_{p}^{(l)}(T) \leq C$, where $l \in\{1,2, \ldots, n\}, p>n / l$ and $1<q \leq l p / n$

(ii) $\mathbf{p}(G) \supset M$ and $\partial T=0$.

As before $A, B, C$ are fixed positive constants, while $M$ is a given $n$-dimensional rectifiable subset of $\mathbf{R}_{x}^{N}$.

Proposition 8.2. The functionals $\mathcal{F}^{\prime}$ and $\mathcal{F}^{\prime \prime}$ defined in $\mathcal{D}$ by

$$
\mathcal{F}^{\prime}(T):=\int \gamma_{T} d\left|T_{(0)}\right|, \quad \mathcal{F}^{\prime \prime}(T):=\int||\left|A_{T}\right||| d\left|T_{(0)}\right|
$$

(see Section 7) have a minimizer.

Proof. We will prove only the assertion about $\mathcal{F}^{\prime}$ in that the same argument works also for $\mathcal{F}^{\prime \prime}$. As in the first application one can obviously assume

$$
\inf \mathcal{F}^{\prime}<+\infty \text {. }
$$

Then, from a minimizing sequence $\left\{T_{j}\right\}$, we can extract a subsequence $\left\{T_{j^{\prime}}\right\}$ such that

$$
T_{j^{\prime}} \rightarrow T \in \operatorname{curv}_{\mathrm{n}}^{*}\left(\mathbf{R}_{x}^{N}\right)
$$

by Proposition 7.5 and the compactness theorem [13, Corollary 4.2]. Moreover $T$ satisfies (i) above, for the semicontinuity theorem [14, Theorem 4.2], while (ii) is fulfilled by [12, Proposition 5.1]. So we get $T \in \mathcal{D}$ and the conclusion follows from Proposition 7.1.

8.3. Third application. Given a couple of real numbers $p, q$ such that $p>n$ and $1<q \leq 2 p / n$, consider the set

$$
\mathcal{D}:=\left\{\varphi \in \operatorname{Imm}^{2, p}\left(V, \mathbf{R}_{x}^{n+1}\right) \mid \operatorname{Area}(\varphi) \leq A, E_{q}^{(1)}\left(T_{\varphi}\right) \leq B\right\}
$$

where, as usual, $A$ and $B$ are positive constants. Then introduce the functional defined in $\operatorname{curv}_{\mathrm{n}}\left(\mathbf{R}_{x}^{n+1}\right)$ by

$$
\mathcal{F}(T):= \begin{cases}E_{p}^{(2)}\left(T_{\varphi}\right)=2^{-p} \int_{V}|\operatorname{Rm}(\varphi)|^{p} & \text { if } T=T_{\varphi} \text { with } \varphi \in \mathcal{D} \\ +\infty & \text { otherwise }\end{cases}
$$


and denote by $\overline{\mathcal{F}}$ the relaxed functional of $\mathcal{F}$ with respect to the weak topology of currents, i.e.,

$$
\overline{\mathcal{F}}=\inf \left\{\liminf _{j} \mathcal{F}\left(T_{j}\right) \mid T_{j} \in \operatorname{curv}_{\mathbf{n}}\left(\mathbf{R}_{x}^{n+1}\right), T_{j} \rightarrow T\right\} .
$$

As a general reference about relaxation we recommend [7].

Proposition 8.3. The following facts hold:

1) if $\overline{\mathcal{F}}(T)<+\infty$, then $T \in \operatorname{curv}_{\mathrm{n}}^{*}\left(\mathbf{R}_{x}^{n+1}\right)$

2) one has $\overline{\mathcal{F}}\left(T_{\varphi}\right)=\mathcal{F}\left(T_{\varphi}\right)$ for all $\varphi \in \mathcal{D}$.

Proof. (1) A sequence $\left\{T_{j}\right\} \subset \operatorname{curv}_{\mathrm{n}}\left(\mathbf{R}_{x}^{n+1}\right)$ has to exist such that

$$
T_{j} \rightarrow T \quad \text { and } \quad \sup _{j} \mathcal{F}\left(T_{j}\right)<+\infty .
$$

Then, by definition of $\mathcal{F}$, we get $T_{j}=T_{\varphi_{j}}$ with $\left\{\varphi_{j}\right\} \subset \mathcal{D}$, which implies

$$
T_{j} \in \operatorname{curv}_{\mathrm{n}}^{*}\left(\mathbf{R}_{x}^{n+1}\right)
$$

by $[\mathbf{1 4}$, Corollary 5.5]. On the other hand, Proposition $7.5(l=2)$ yields

$$
\sup _{j} \mathcal{G}_{F_{q}}\left(T_{j}\right)<+\infty
$$

and hence, recalling the compactness theorem [13, Corollary 4.2], we conclude that $T$ is a special generalized Gauss graph.

(2) It follows at once from the lowersemicontinuity of $E_{p}^{(2)}[\mathbf{1 4}$, Theorem $4.2]$.

\section{References}

[1] M.T. Anderson, Degeneration of metrics with bounded curvature and applications to critical metrics of Riemannian functionals, Proc. Symp. Pure Math., 54 (1993), Part 3, 53-79.

[2] G. Anzellotti, Functionals depending on curvatures, Rend. Sem. Mat. Univ. Politec., Torino, Fascicolo speciale 1989; P.D.E. and Geometry, (1988), 47-62.

[3] G. Anzellotti and S. Delladio, Minimization of Functionals of Curvatures and the Willmore Problem, Advances in Geometric Analysis and Continuum Mechanics, International Press, (1995), 33-43.

[4] G. Anzellotti and R. Serapioni, $\mathcal{C}^{k}-$ rectifiable sets, J. Reine Angew. Math., 453 (1994), 1-20.

[5] G. Anzellotti, R. Serapioni and I. Tamanini, Curvatures, functionals, currents, Indiana Univ. Math. J., 39 (1990), 617-669.

[6] Yu.D. Burago and V.A. Zalgaller, Geometric Inequalities, Grundlehren der mathematischen Wissenschaften, 285, Springer-Verlag, 1988.

[7] G. Buttazzo, Semicontinuity, Relaxation and Integral Representation in the Calculus of Variations, Longman, Harlow, 1989.

[8] C. Castaing and M. Valadier, Convex Analysis and Measurable Multifunctions, Lecture Notes in Mathematics, 580, Springer-Verlag, 1977. 
[9] I. Chavel, Riemannian Geometry: A Modern Introduction, Cambridge Tracts in Mathematics, 108, Cambridge University Press, 1995.

[10] S. Delladio, Slicing of Generalized Surfaces with Curvatures Measures and Diameter's Estimate, Annales Polonici Mathematici, LXIV.3 (1996), 267-283.

[11] _ Do Generalized Gauss Graphs Induce Curvature Varifolds?, Boll. Unione Matematica Italiana, 10-B (1996), 991-1017.

[12] _ Minimizing functionals on surfaces and their curvatures: A class of variational problems in the setting of generalized Gauss graphs, Pacific J. Math., 179 (1997), 301-323.

[13] Special generalized Gauss graphs and their application to minimization of functionals involving curvatures, J. Reine Angew. Math., 486 (1997), 17-43.

[14] On hypersurfaces in $\mathbf{R}^{n+1}$ with integral bounds on curvature, to appear on J. Geom. Anal.

[15] S. Delladio and G. Scianna, Oriented and nonoriented curvature varifolds, The Royal Society of Edinburgh Proceedings, 125A (1995), 63-83.

[16] M.P. Do Carmo, Differential Geometry of Curves and Surfaces, Prentice-Hall, Inc., Englewood Cliffs, New Jersey, 1976.

[17] _ Riemannian Geometry, Birkhäuser, Boston, 1992.

[18] H. Federer, Geometric Measure Theory, Springer-Verlag, 1969.

[19] J.E. Hutchinson, Second fundamental form for varifolds and the existence of surfaces minimizing curvature, Indiana Univ. Math. J., 35 (1986), 45-71.

$[20] \_, C^{1, \alpha}$ multiple function and tangent cone behaviour for varifolds with second fundamental form in $L^{p}$, Proc. Sympos. Pure Math., 44 (1986), 281-306.

[21] Some regularity theory for curvature varifolds, Proc. Center Math. Analysis, Australian National University, 12 (1987), 60-66.

[22] _ Poincare-Sobolev and related inequalities for submanifolds of $\mathbf{R}^{\mathbf{n}}$, Pacific Journal of Math., 145 (1990), 59-69.

[23] J. Langer, A compactness theorem for surfaces with $L_{p}$-bounded second fundamental form, Mathematische Annalen, 270 (1985), 223-234.

[24] C. Mantegazza, Curvature Varifolds with boundary, to appear on Journal of Differential Geometry.

[25] M. Marcus, Finite Dimensional Multilinear Algebra, Part II, Marcel Dekker, Inc., New York, 1975.

[26] F. Morgan, Geometric Measure Theory, a beginner's guide, Academic Press Inc., 1988.

[27] W. Rudin, Real and Complex Analysis, McGraw-Hill, 1970.

[28] L. Simon, Lectures on Geometric Measure Theory, Proceedings of the Centre for Mathematical Analysis, Canberra, Australia, Vol. 3, 1984.

Dipartimento Di Matematica

UNIVERSITÀ DI TRENTO

38050 Povo

TREnTo

ITALY

E-mail address: delladio@science.unitn.it 